\title{
0 papel mediador dos estilos parentais no ajustamento psicológico de crianças e adolescentes com doenças crônicas
}

\author{
Amanda Muglia Wechsler \\ Denise Catricala Bution \\ Taís Galvão \\ Jaqueline Garcia Diniz \\ Cristiane Bossi Gabriel
}

\section{RESUMO}

Uma doença crônica infantil representa uma grande fonte de stress para os pacientes e suas famílias. O presente estudo objetivou avaliar o ajustamento psicológico de crianças/adolescentes com doenças crônicas e de seus cuidadores, assim como o papel mediador dos estilos parentais. Participaram desta pesquisa 41 crianças/adolescentes com enfermidades crônicas e 72 crianças "saudáveis" e seus respectivos pais/cuidadores. Os resultados mostraram que os grupos não diferiram entre si com relação ao ajustamento psicológico tanto das crianças/adolescentes quanto dos cuidadores. Também se constatou que a negligência e punição inconsistente mediaram a relação entre o desajustamento infantil e o parental. Conclui-se sobre a natureza multideterminada e multidirecional do ajustamento psicológico, assim como a importância de intervenções psicológicas hospitalares que promovam práticas parentais positivas.

Palavras-chave: Doenças crônicas; Hospitalização; Estilos parentais; Ajustamento psicológico; Psicologia Hospitalar

\section{ABSTRACT}

The mediating role of parental styles in the psychological adjustment of chronic ill children and adolescents

A chronic childhood illness represents a great source of stress to patients and their families. The objective of the present study was to evaluate the psychological adjustment of children/ adolescents with chronic diseases and their caregivers and verify the mediating role of parental styles. 41 children/adolescents with chronic diseases and 72 healthy children and their respective parents/caregivers participated in this study. The groups did not differ concerning the psychological adjustment of both the children and their caregivers. It was also found that negligence and inconsistent punishment mediated the relationship between children and parental maladjustment. Conclusions highlight the psychological adjustment multidetermined and multidirectional nature, as well as the importance of psychological interventions aimed at promoting positive parental practices.

Keywords: Chronic Diseases; Hospitalization; Parenting Styles; Psychological Adjustment; Health Psychology.

Uma doença crônica é uma condição médica não-transmissível, de longa duração, lenta progressão e raramente prevê uma cura completa (Organização Mundial da Saúde, 2014). No Brasil, por volta de $10 \%$ da população infanto-juvenil possui alguma doença crônica, representando uma grande fonte de stress para a criança e para a sua família (Ministério da Saúde, 2014). 0 diagnóstico e tratamento destas enfermidades provocam alterações na rotina diária, dor e medo

\section{Sobre os autores}

A. M. W. http://orcid.org/0000-0001-

5916-1667

Pontifícia Universidade Católica de Campinas - Campinas, SP amanda_wechsler@yahoo.com. br

D. C. B

https://orcid.org/0000-0002-

0285-1269

Centro Universitário Unifafibe -

Bebedouro, SP

dbution@gmail.com

T. G.

https://orcid.org/0000-0003-

2072-4834

Faculdade de Ciências e Saúde de Barretos - Barretos, SP tais.galvao@hotmail.com

J. G. D.

https://orcid.org/0000-0002-

3080-3221

Centro Universitário Estácio de Ribeirão Preto - Ribeirão Preto, SP

psicojack.brain@gmail.com

C. B. G. https://orcid.org/0000-00034024-4010

Associação de Pais e Amigos dos Excepcionais -APAE - Bebedouro, SP

E-mail: cristianebg_@hotmail. com

\section{Direitos Autorais}

Este é um artigo de acesso aberto e pode ser reproduzido livremente, distribuído, transmitido ou modificado, por qualquer pessoa desde que usado sem fins comerciais. 0 trabalho é disponibilizado sob a licença Creative Commons CC-BY-NC.

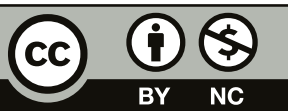


dos procedimentos médicos, disfunções físicas e/ou cognitivas, estigma social e ansiedade relacionada à incerteza sobre a doença, seu tratamento e prognóstico (Compas et al., 2012).

Algumas pesquisas sinalizam que, em função da exposição a uma grande quantidade de estressores, as crianças com doenças crônicas parecem ter maior risco de apresentar problemas de ajustamento psicológico do que crianças "saudáveis" (Bai et al., 2017; Ferro \& Boyle, 2013; Pinquart, 2011). Assim, estas crianças apresentariam mais problemas internalizantes (por exemplo, ansiedade, depressão, retraimento) e externalizantes (agressividade, impulsividade, entre outros) do que seus pares. No entanto, outras pesquisas não encontraram evidências significativas de disfunções psicológicas e sociais em pacientes pediátricos com enfermidades crônicas, e a maioria deles apresentava considerável resiliência (Barros et al., 2008; Ferguson \& Walker, 2012).

Deste modo, a literatura científica ainda não parece ter claras as fontes de variação individual das diferenças de ajustamento psicológico entre crianças com doenças crônicas, incluindo os fatores que diferenciam as crianças resilientes das que se encontram em risco psicológico (Santos et al., 2013). Neste sentido, alguns estudos sugerem que variáveis médicas seriam preditoras fracas e que o funcionamento familiar, a relação pais-filhos e o ajustamento parental seriam preditores mais potentes, visto que a condição crônica de saúde teria impacto em todo o sistema familiar (Cousino \& Hazen, 2013; Ferro \& Boyle, 2015; Fee \& Hinton, 2011; Jackson et al., 2015).

Por outro lado, Leinonen et al. (2003) e Nabors et al. (2013) sugerem que $o$ ajustamento parental ou as práticas parentais podem não atuar diretamente sobre o ajustamento psicológico das crianças, funcionando mais como mediadores. Mediadores, segundo Holmbeck (1997), seriam variáveis afetadas pela variável independente e que, por sua vez, influenciariam na variável dependente. Assim, a relação de interesse do presente estudo entre variável independente (doenças crônicas) e dependente (ajustamento psicológico infantil) não seria direta, mas sim mediada pelas práticas parentais.

Em seu conjunto, as práticas parentais podem ser definidas como um estilo parental. 0 estilo parental positivo abrange a monitoria positiva e o comportamento moral. Já o estilo parental negativo envolve práticas educativas de negligência, abuso físico e/ou psicológico, disciplina relaxada, punição inconsistente e monitoria negativa (Gomide, 2006).

Neste sentido, algumas revisões de literatura destacam que crianças com doenças crônicas estão submetidas a mais práticas parentais educativas negativas do que seus pares (Murphy et al., 2017; Pinquart, 2017; Santos et al., 2013), o que as colocaria em maior risco de sofrer problemas psicológicos. Além disto, práticas parentais negativas nesta população estão relacionadas a uma menor adesão aos tratamentos mé- dicos, menor qualidade de vida e piores indicadores de saúde (Ellis et al., 2007; Jaser \& Grey, 2010; Olvera \& Power, 2010).

Portanto, observa-se a necessidade de identificar os processos associados com resultados tão diversos expostos na literatura sobre a qualidade do ajustamento psicológico de crianças com doenças crônicas. Além disto, urgem estudos sobre estilos parentais no ajustamento de crianças e adolescentes com doenças crônicas brasileiras e seu papel mediador, visto que publicações internacionais sobre o tema são escassas e nacionais quase inexistentes (Piccinini et al., 2003).

Consequentemente, o presente estudo teve como objetivo comparar o ajustamento psicológico de crianças e adolescentes com doenças crônicas com o de crianças sem este histórico. Ademais, visou-se avaliar se o ajustamento psicológico de seus cuidadores exercia influência no ajustamento das crianças. Por último, foi verificado o papel mediador dos estilos parentais na relação entre ajuste psicológico dos cuidadores e das crianças.

As hipóteses desta pesquisa foram: 1) As crianças/adolescentes com enfermidades crônicas e seus cuidadores possuiriam menor ajustamento psicológico que crianças/ adolescentes "saudáveis" e seus cuidadores; 2) 0 ajustamento psicológico das crianças/adolescentes estaria diretamente relacionado ao ajustamento psicológico de seus cuidadores; 3) Práticas parentais positivas e negativas mediariam o ajustamento psicológico das crianças/adolescentes e de seus cuidadores.

\section{MÉTODO}

\section{PARTICIPANTES}

0 presente estudo, com delineamento descritivo transversal, utilizou-se de uma amostra de 131 crianças e adolescentes (8-14 anos de idade, $52,7 \%$ do sexo masculino) e seus respectivos pais/cuidadores, divididos em dois grupos: clínico (com doenças crônicas) e controle (sem doenças crônicas).

No grupo clínico (GCL), foi utilizada uma amostra de conveniência de 41 crianças e adolescentes com doenças crônicas e seus respectivos cuidadores, dentre 45 pacientes abordados pelas pesquisadoras. Os participantes foram recrutados em dois hospitais gerais situados no estado de São Paulo. Os principais motivos alegados pelos cuidadores para não participar da pesquisa foram falta de tempo ou desinteresse no tema. Foram excluídas crianças/adolescentes com câncer, com doenças terminais ou em cuidados paliativos, que não estivessem alfabetizadas ou que apresentassem alguma comorbidade neurológica ou psiquiátrica que as impedia de responder aos questionários. Assim, apesar de doenças neurológicas terem sido incluídas como doenças crônicas no grupo clínico, 
foram excluídas quando eram comórbidas a outras doenças crônicas. Como critério de inclusão dos cuidadores, estes deveriam ser o principal cuidador da criança (pai, mãe, avô, avó, tia ou tio).

No grupo controle (GCO), participaram 72 crianças/adolescentes sem histórico de doença crônica e seus respectivos cuidadores. Este grupo foi recrutado em quatro escolas públicas situadas nas mesmas cidades dos hospitais, através do envio de carta convite a 477 responsáveis pelas crianças/adolescentes. Destes, setenta e dois responderam, aceitando participar, possuindo idades e nível socioeconômico similares ao GCL. As crianças deste grupo não poderiam ter tido nenhum histórico de doença crônica física ou mental e deveriam estar alfabetizadas. 0 mesmo critério de inclusão dos cuidadores do GCL foi adotado.

\section{INSTRUMENTOS}

Questionário Sóciodemográfico e Médico. Os dados sociodemográficos e médicos dos participantes foram obtidos a partir de questionário "ad hoc" desenvolvido pelas pesquisadoras. Tal questionário foi respondido pelos cuidadores e contemplava informações relativas a idade, sexo, diagnóstico e tempo em tratamento das crianças; além de escolaridade, idade, religião, estado civil e renda do cuidador.

Child Behavior Checklist (CBCL) - adaptado e validado por Silvares et al. (2001). Este inventário mede comportamentos-problema e adaptativos em crianças e adolescentes e possui 138 itens, para os quais o respondente deve marcar a frequência com que esses problemas ocorrem, a partir de uma escala de 0 a 2. A aplicação do formulário é realizada com os responsáveis de crianças e adolescentes entre 6 e 18 anos, e avalia problemas de comportamentos internalizantes, externalizantes e totais, sendo os três indicadores incluídos no presente estudo. O comportamento da criança é classificado como "clínico", "limítrofe" ou "não-clínico", de acordo com a amostra normativa. A precisão por alfa de Cronbach da versão brasileira deste teste varia de 0,83 a 0,95 nas escalas gerais (Achenbach \& Rescorla, 2007). No presente estudo, o alfa de Cronbach foi de 0,93 para a pontuação total, 0,74 para a dimensão de problemas externalizantes e 0,72 para problemas internalizantes.

Escala de Stress Infantil (ESI) - Lipp e Lucarelli (2005). Este instrumento avalia quatro dimensões do stress infantil (reações físicas, psicológicas, psicológicas com componente depressivo e psicofisiológicas) a partir de 35 itens em escala likert de 0 a 4 pontos. Neste estudo, foi considerado o escore total, referente à soma destas quatro dimensões. Escores acima de 79,40 na escala total indicam níveis clínicos de estresse (fases de quase-exaustão ou exaustão). É apropriado para crianças e adolescentes de 6 a 14 anos, e tem consistência interna de
0,89 no estudo original (Lipp \& Lucarelli, 2005) e de 0,83 com a presente amostra.

Escala de Autoconceito Infanto-Juvenil (EAC-IJ) - Sisto e Martinelli (2004). Esta escala mede o autoconceito de crianças e adolescentes de 8 a 16 anos, em quatro dimensões: autoconceito pessoal, social, familiar e escolar. Está composta de 20 itens, dispostos em escala likert de 0 a 3 pontos. No presente estudo, foi considerado o escore total, referente à soma destas quatro dimensões. Um baixo autoconceito total (quartil 25) está relacionado a pontuações menores de 22 para a faixa etária de 8 a 14 anos. 0 coeficiente de consistência interna de Cronbach é de 0,78 para a pontuação total no estudo original (Sisto \& Martinelli, 2004) e de 0,64 na presente pesquisa.

Inventário Beck de Depressão - II (BDI-II) - adaptado e validado por Cunha (2001). O Inventário Beck de Depressão mede a presença e intensidade de sintomas depressivos em adultos. Tal instrumento contém 21 itens, graduados de 0 a 3 . Na versão brasileira, uma pontuação menor ou igual a 11 pontos indica ausência de depressão; entre 12 e 19 se considera que há depressão leve; entre 20 a 35 depressão moderada e acima disto, depressão grave. A consistência interna do instrumento é de 0,82 tanto no estudo original (Cunha, 2001) quanto na presente pesquisa.

Inventário Beck de Ansiedade (BAI) - adaptado e validado por Cunha (2001). O Inventário BAI mede a ansiedade física, cognitiva e afetiva do indivíduo através de 21 itens, dispostos em formato likert ( 0 a 3 pontos). Considera-se que de 0 a 9 pontos não há indicativos de ansiedade, de 10 a 19 há ansiedade leve, de 20 a 30 ansiedade moderada e acima deste valor, ansiedade severa. A consistência interna da versão brasileira é de 0,90 segundo o manual do instrumento (Cunha, 2001) e de 0,89 no presente estudo.

Bateria Fatorial de Personalidade (BFP) - Nunes, Hutz e Nunes (2013). Este instrumento mede traços de personalidade de adultos em cinco dimensões: Extroversão, Socialização, Realização, Neuroticismo e Abertura. A bateria contém 126 itens, dispostos em uma escala likert, com graus que variam de 1 a 7. Nesta pesquisa, foi utilizada somente a subescala de Neuroticismo, cujo alfa de Cronbach é de 0,89 no estudo original (Nunes et al., 2013) e de 0,82 com a presente amostra.

Inventário de Estilos Parentais (IEP) - Gomide (2002). Este inventário mede o conjunto de práticas educativas utilizadas pelos pais na interação com os filhos em sete dimensões (abuso físico, punição inconsistente, disciplina relaxada, monitoria negativa, negligência, monitoria positiva e comportamento moral) e de forma total. Todas estas dimensões, assim como o escore total, foram considerados na presente pesquisa. 0 instrumento contém 42 questões, dispostas em formato likert de 0 a 2 pontos, e a pontuação total classifica a família como de risco (pontuações entre -8 e -34) ou de não-risco (pontuações 
entre 7 e 15). Sua consistência interna varia de 0,46 a 0,87 entre os fatores no estudo original (Gomide, 2002) e de 0,44 a 0,63 na presente pesquisa.

\section{PROCEDIMENTO}

A presente pesquisa faz parte de um projeto mais amplo, aprovado pelo Comitê de Ética de Pesquisa com Seres Humanos sob o número [CAAE 30409214.5.0000.5387] na Plataforma Brasil. Aplicou-se, em ambos grupos participantes, após a assinatura dos Termos de Consentimento e Assentimento, o ESI e o EAC-IJ nas crianças/adolescentes e os pais/cuidadores preencheram o $\mathrm{CBCL}, \mathrm{BAI}, \mathrm{BDI}$, IEP e BFP, nesta ordem. Esta mesma ordem de aplicação foi seguida com todos os participantes, de forma a não enviesar os resultados em função do cansaço dos sujeitos. Quatro estudantes de Psicologia, que estavam em seu último ano de sua formação, foram treinados para aplicar os questionários nos dois grupos participantes.

Os participantes do GCL foram abordados nos hospitais participantes, enquanto esperavam consultas de rotina. A estes, houve a explicação da pesquisa e posterior assinatura do Termo de Consentimento Livre e Esclarecido e de Assentimento. Os pais/cuidadores e as crianças/adolescentes preencheram os instrumentos individualmente e separadamente em uma sala vazia do hospital. Eventuais dúvidas foram sanadas durante o preenchimento, que durou em torno de 30 minutos.

As crianças/adolescentes do GCO foram abordadas nas escolas públicas nas quais estudavam. Elas receberam uma carta explicativa sobre a pesquisa, juntamente com o Termo de Consentimento e de Assentimento, e foram instruídas a levá-los a seus pais ou cuidadores e devolvê-las ao pesquisador responsável nas semanas subsequentes. Aos pais/cuidadores que devolveram o Termo de Consentimento e de Assentimento assinados, foram enviados os instrumentos psicológicos em um envelope opaco lacrado. Neste envelope também havia telefone e e-mail do pesquisador para possíveis dúvidas. Os pais/cuidadores devolveram os instrumentos preenchidos no envelope lacrado através de seus filhos, e o pesquisador os coletou nas escolas. Já com as crianças/adolescentes, os instrumentos foram aplicados individualmente em uma sala vazia da sua respectiva escola.

\section{ANÁLISE DOS DADOS}

Para a análise univariada dos dados, foram utilizadas a frequência, a média e o desvio padrão. 0 teste $t$ de Student foi usado para comparar os grupos com relação ao ajustamento psicológico infantil e parental e com relação às práticas parentais. Para analisar a relação entre variáveis demográficas e o ajustamento psicológico das crianças/adolescentes, foram empregadas correlações de Pearson, e para avaliar o nível de concordância entre pais e filhos utilizaram-se correlações intraclasse.

A análise dos mediadores foi realizada primeiramente com correlações de Pearson para verificar o cumprimento de critérios nos modelos de regressão de três passos proposto por Baron e Kenny (1986). Todos os modelos propostos foram confirmados tanto pela análise dos coeficientes e dos alfas $(a=0,05)$ em cada passo das regressões, quanto pelas análises post-hoc.

\section{RESULTADOS}

Os dados sociodemográficos da amostra encontram-se descritos na Tabela 1, abaixo. De modo geral, a amostra dos cuidadores foi constituída em sua maioria por mães (87\%) e pais $(10,2 \%)$, de baixo nível socioeconômico, praticantes de alguma religião e casados ou amasiados. Os demais cuidadores eram compostos de avós, tios e irmãos mais velhos (2,8\%). 
Tabela 1 Características Demográficas das Famílias Participantes (Casos Válidos)

\begin{tabular}{|c|c|c|}
\hline Variáveis & Grupo clínico $(n=41)$ & Grupo controle $(n=74)$ \\
\hline \multicolumn{3}{|l|}{ Crianças } \\
\hline \multicolumn{3}{|l|}{ Gênero } \\
\hline Feminino & $18(43,9 \%)$ & $44(59,5 \%)$ \\
\hline Masculino & $23(56,1 \%)$ & $30(40,5 \%)$ \\
\hline Idade - média (DP) & $11,39(1,87)$ & $10,53(1,95)$ \\
\hline \multicolumn{3}{|l|}{ Cuidadores } \\
\hline Idade - média (DP) & $38,80(7,39)$ & $35,80(5,80)$ \\
\hline \multicolumn{3}{|l|}{ Escolaridade } \\
\hline Ensino fundamental & $21(51,2 \%)$ & $27(36,5 \%)$ \\
\hline Ensino médio/superior & $20(31,7 \%)$ & $46(41,9 \%)$ \\
\hline \multicolumn{3}{|l|}{ Religião } \\
\hline Com religião & $40(97,6 \%)$ & $69(93,2 \%)$ \\
\hline Sem religião & $1(2,4 \%)$ & $5(6,8 \%)$ \\
\hline Variáveis & Grupo clínico $(n=41)$ & Grupo controle $(n=74)$ \\
\hline \multicolumn{3}{|l|}{ Estado civil } \\
\hline Casado/amasiado & $31(75,6 \%)$ & $58(78,4 \%)$ \\
\hline Solteiro/separado/viúvo & $10(24,4 \%)$ & $16(21,6 \%)$ \\
\hline Renda familiar bruta - média (DP) (em reais) & $1815,05(887,72)$ & $1926,86(1048,23)$ \\
\hline
\end{tabular}

O diagnóstico mais presente no GCL era o de doenças respiratórias (asma, bronquite e rinite), representando $68,3 \%$ da amostra. 0 segundo diagnóstico mais comum era o de doenças neurológicas (paralisia cerebral, AVC, epilepsia e cisto aracnoide), com 12,2\%. Doenças endocrinológicas (diabetes e dislipidemia) e outras doenças (hipertensão e doença renal crônica) estavam presentes em 7,3\% das crianças, cada. Por último, doenças degenerativas (distrofia muscular e fibromatose) representavam $4,9 \%$ da amostra. 0 tempo médio em tratamento era de 5,5 anos $(\mathrm{DP}=3,52)$ até o momento do recrutamento.

No GCL, 7,3\% das crianças/adolescentes demonstrou ter problemas psicológicos em níveis clínicos e $22 \%$ apresen- tou baixo autoconceito geral. Quanto aos cuidadores, $14,6 \%$ apresentou ansiedade, $2,4 \%$ relatou sintomas depressivos em níveis clínicos (depressão moderada ou grave) e 41,5\% apresentou um estilo parental geral de risco. No GCO, 5,4\% das crianças/adolescentes apresentou desajustamento psicológico, $28,4 \%$ relatou baixo autoconceito e $1,1 \%$ tinha níveis clínicos de stress. Em relação aos cuidadores, 9,72\% tinha níveis clínicos de ansiedade e depressão e 20,5\% apresentou pontuações consideradas de risco em seus estilos parentais.

A Tabela 2 evidencia as correlações entre os problemas de ajustamento das crianças/adolescentes e o de seus cuidadores. Observam-se correlações significativas principalmente no GCL. 
Tabela 2: Correlações entre Problemas de Ajustamento de Crianças/Adolescentes e Seus Pais/Cuidadores nos Dois Grupos Participantes (Casos Válidos)

\begin{tabular}{|c|c|c|c|c|c|c|}
\hline \multirow{3}{*}{ Problemas de ajustamento infantil } & \multicolumn{6}{|c|}{ Problemas de ajustamento dos pais/cuidadores } \\
\hline & \multicolumn{3}{|c|}{ Grupo clínico $(\mathrm{n}=41)$} & \multicolumn{3}{|c|}{ Grupo controle $(n=74)$} \\
\hline & Depres & Ansied & Neurot. & Depres. & Ansied. & Neurot. \\
\hline Cptos-probl.-total (CBCL) & $0,448 * \star$ & $0,463^{\star \star}$ & $0,356^{\star}$ & $0,308 * *$ & $0,279 *$ & 0,186 \\
\hline External. problemas (CBCL) & $0,369 *$ & $0,440 * \star$ & 0,263 & $0,330 \star \star$ & $0,277^{*}$ & 0,184 \\
\hline Internal. problemas (CBCL) & $0,393^{*}$ & $0,375^{\star}$ & $0,327^{\star}$ & $0,240 *$ & 0,189 & 0,146 \\
\hline Stress infantil - total (ESI) & 0,229 & $0,322 *$ & $0,373^{*}$ & 0,155 & 0,174 & 0,171 \\
\hline
\end{tabular}

Depres. $=$ depressão (BDI); Ansied= ansiedade (BAI); Neurot.= neuroticismo (BFP). Cptos-probl. total $(\mathrm{CBCL})=$ total de comportamentos-problema infanto-juvenis no $\mathrm{CBCL}$; External. problemas = externalização de problemas; Internal. problemas= internalização de problemas. ${ }^{*} p<0,05 ;{ }^{*} \mathrm{p}<0,01$.

Destaca-se a presença de correlações positivas e significativas entre problemas de ajustamento dos cuidadores e das crianças do GCL, com exceção da escala de neroticismo para problemas externalizantes e de sintomas depressivos para o escore total de stress infantil. No GCO, maiores escores de depressão e ansiedade estiveram relacionados a maiores pontuações de problemas de comportamento infantil. Especificamente, observam-se problemas externalizantes e internalizantes das crianças do GCO quando conviviam com cuidadores com maiores escores de depressão.
Por outro lado, a Tabela 3 compara o ajustamento psicológico de crianças/adolescentes e de seus pais nos dois grupos, mostrando que os grupos não diferiram entre si com relação ao ajustamento psicológico tanto das crianças quanto de seus pais/cuidadores. Pôde-se observar uma diferença entre grupos marginalmente significativa $(p=0,060)$ nos estilos parentais (total), apesar de tal diferença não ser constatada nas subescalas deste inventário. Mesmo quando os participantes com doenças menos graves (respiratórias) foram retirados das análises, os resultados foram mantidos.

Tabela 3: Comparações entre Grupos - Variáveis de Ajustamento Psicológico e Mediadoras para as Crianças/ Adolescentes e para os Pais/ Cuidadores

\begin{tabular}{|c|c|c|c|c|c|}
\hline \multirow{2}{*}{$\begin{array}{l}\text { Ajustamento infantil e } \\
\text { pais/cuidadores }\end{array}$} & \multicolumn{2}{|c|}{ Grupo clínico $(\mathrm{n}=41)$} & \multicolumn{2}{|c|}{ Grupo controle $(n=74)$} & \multirow{2}{*}{$\mathrm{p}$} \\
\hline & Média & $\mathrm{DP}$ & Média & $\mathrm{DP}$ & \\
\hline Cptos-problema - total (CBCL) & 40,90 & 22,76 & 33,82 & 21,55 & 0,103 \\
\hline Stress infantil - total (ESI) & 8,92 & 4,82 & 7,59 & 4,23 & 0,130 \\
\hline Autoc. infantil - total (EAC-IJ) & 24,22 & 6,00 & 25,20 & 4,36 & 0,315 \\
\hline Depressão (BDI) & 9,67 & 9,08 & 8,51 & 8,43 & 0,496 \\
\hline Ansiedade (BAI) & 7,95 & 6,37 & 9,72 & 7,82 & 0,221 \\
\hline Neurotic. - total (BFP) & 3,39 & 0,81 & 3,51 & 1,30 & 0,570 \\
\hline Estilos parentais - total (EAP) & 35,95 & 8,44 & 38,82 & 6,15 & 0,060 \\
\hline
\end{tabular}

DP = desvio-padrão; Cptos-problema - total= total de comportamentos-problema infanto-juvenis no CBCL; Autoc. Infantil= autoconceito infanto-juvenil; Neurotic. $=$ neuroticismo.

Tanto os cuidadores quanto as próprias crianças/adolescentes com doenças crônicas mostraram concordância ao relatar os problemas de ajustamento infantis. As correlações intraclasse entre os comportamentos-problema infantis e o stress infantil total no GCL foram estatisticamente significati$\operatorname{vas}(r=0,546 ; p=0,003)$. No entanto, tal resultado não foi encontrado no GCO $(r=0,305 ; p=0,066)$.
Ao analisar as variáveis demográficas, constatou-se que as crianças/adolescentes do GCL que haviam sido diagnosticadas há mais tempo relatavam um menor autoconceito geral $(r=-0,428 ; p=0,016)$, e as que possuíam pais/cuidadores com idades mais elevadas apresentavam mais problemas de ajustamento $(r=0,432 ; p=0,006)$. No GCO, uma menor idade da 
criança/adolescente estava associada a maior autoconceito geral $(r=-0,292 ; p=0,012)$. Para os cuidadores de ambos os grupos, nenhuma variável sociodemográfica esteve correlacionada significativamente às suas medidas de ajustamento psicológico.

Com relação às práticas parentais adotadas (monitoria positiva: $p=0,226$; comportamento moral: $p=0,994$; punição inconsistente: $p=0,078$; negligência: $p=0,496$; disciplina relaxada: $p=0,183$; monitoria negativa: $p=0,169$; abuso físico: $p=0,626$ ), não houve diferenças significativas entre os grupos participantes.

Para avaliar se os estilos parentais atuavam como mediadores, foram executadas correlações preliminares entre as variáveis dependentes (CBCL, ESI e EAC-IJ) e as independentes (BDI, BAI e BFP) e o mediador (IEP). Tais dados estão descritos na Tabela 4.

Tabela 4: Matriz Correlacional para Crianças do Grupo Clínico e do Grupo Controle

\begin{tabular}{|c|c|c|c|c|c|c|}
\hline Variáveis & Stress inf. & Autoconceito inf. & Depressão par. & Ansiedade par. & $\begin{array}{l}\text { Neuroticismo } \\
\text { par. }\end{array}$ & Estilos par. \\
\hline \multirow{2}{*}{$\begin{array}{l}\text { Cptos-proble- } \\
\text { ma infantis }\end{array}$} & 0,251 & $-0,151$ & $0,448^{\star \star}$ & $0,462^{\star \star}$ & $0,356^{*}$ & $0,445^{\star \star}$ \\
\hline & 0,144 & $-0,200$ & $0,279 *$ & $0,285^{\star}$ & $0,308^{\star *}$ & $0,295^{\star}$ \\
\hline \multirow{2}{*}{ Stress infantil } & & $-0,164$ & 0,110 & 0,115 & 0,073 & 0,061 \\
\hline & & $-0,452 * \star$ & 0,157 & $0,231 *$ & 0,206 & 0,044 \\
\hline \multirow{2}{*}{$\begin{array}{l}\text { Autoconceito } \\
\text { infantil }\end{array}$} & & & $-0,223$ & $-0,001$ & $-0,244$ & $-0,142$ \\
\hline & & & $-0,162$ & $-0,131$ & $-0,237^{\star}$ & $-0,085$ \\
\hline \multirow{2}{*}{$\begin{array}{l}\text { D e p res são } \\
\text { pais/cuidado- } \\
\text { res }\end{array}$} & & & & $0,647 * \star$ & 0,313 & $0,372^{*}$ \\
\hline & & & & $0,570 * *$ & $0,563^{\star \star}$ & $0,236^{*}$ \\
\hline \multirow{2}{*}{$\begin{array}{l}\text { A n siedade } \\
\text { pais/cuidado- } \\
\text { res }\end{array}$} & & & & & $0,348^{*}$ & 0,254 \\
\hline & & & & & $0,248^{*}$ & 0,143 \\
\hline \multirow{2}{*}{$\begin{array}{l}\text { Neuroticismo } \\
\text { pais/cuidado- } \\
\text { res }\end{array}$} & & & & & & $0,552^{\star *}$ \\
\hline & & & & & & $0,299 *$ \\
\hline
\end{tabular}

${ }^{*} \mathrm{p}<0,05 ;{ }^{*} \mathrm{p}<0,01$. Inf $=$ infantil; par. $=$ parental. Cptos-problema - total $=$ total de comportamentos-problema infantis no CBCL A primeira linha de cada célula corresponde ao grupo clínico, e a segunda linha ao grupo controle.

Os resultados descritos na Tabela 4 indicam que os comportamentos-problema infanto-juvenis estavam significativamente relacionados tanto com o ajustamento dos pais/ cuidadores (variáveis independentes) quanto com os estilos parentais. Por sua vez, o stress e o autoconceito infanto-juvenis não estavam relacionados às variáveis independentes e nem à mediadora. Deste modo, o modelo proposto examinou o impacto do ajustamento psicológico dos pais/cuidadores (ansiedade, depressão e neuroticismo) no ajustamento infanto-juvenil medido através do teste $\mathrm{CBCL}$ (comportamentos-problema infanto-juvenis) nos dois grupos participantes.

Assim, foi adotado um modelo de regressão sem as variáveis "stress infantil" e autoconceito infantil". O primeiro passo da regressão confirmou que os preditores se relacionavam de forma significativa com os estilos parentais no GCL em relação à depressão $(\beta=0,372 ; E P B=0,201 ; p=0,018)$ e neuroticismo $(\beta=0,552 ; E P B=1,409 ; p<0,0001)$, mas não em ansiedade $(\beta=0,254 ; E P B=0,147 ; p=0,113)$ dos pais/cuidadores. No GCO também se observaram as mesmas relações em depressão $(\beta=0,236 ; \mathrm{EPB}=0,092 ; p=0,046)$ e neuroticismo $(\beta=0,299$; $\mathrm{EPB}=0,562 ; \mathrm{p}=0,013)$, mas não em ansiedade $(\beta=0,143$; $\mathrm{EPB}=$ 0,$085 ; p=0,228$ ) dos pais/cuidadores.

No segundo passo da regressão, ao excluir a variável "ansiedade", verificou-se que o ajustamento infantil estava relacionado às duas medidas de ajustamento dos pais/cuidadores, tanto no grupo clínico (depressão: $\beta=0,448$; $E P B=0,519 ; p=$ 0,004 / neuroticismo: $\beta=0,356$; $E P B=4,373 ; p=0,026$ ) quanto no grupo controle (depressão: $\beta=0,279 ; E P B=0,317 ; p=0,017$ / 


\section{INTERACÃO EM E. PSICOLOGIA}

Amanda Muglia Wechsler, Denise Catricala Bution, Taís Galvão, Jaqueline Garcia Diniz e

Cristiane Bossi Gabriel

neuroticismo: $\beta=0,308 ; E P B=1,945 ; p=0,010$ ).

0 terceiro e último passo da regressão está descrito na Tabela 5 , abaixo, que descreve o papel mediador dos estilos parentais entre o ajustamento psicológico dos pais/cuidadores e o ajustamento psicológico infantil. Pode-se observar nesta tabela que a relação entre os preditores (BDI e BFP) e a variável dependente (CBCL) é menor no GCL quando o mediador está na equação, o que indica o efeito mediador dos estilos parentais. No GCO, não se observaram tais efeitos. A análise post-hoc confirmou o efeito mediador dos estilos parentais no GCL entre os comportamentos-problema infantis e a depressão dos pais /cuidadores (teste Sobel $=1,931 ; p=0,050$ ) e entre o neuroticismo dos pais/cuidadores e os comportamentos-problema das crianças (teste Sobel=2,486; $p=0,0120$ ).

Tabela 5: Papel Mediador dos Estilos Parentais entre o Ajustamento Psicológico dos Pais/Cuidadores e o Ajustamento Psicológico Infantil

\begin{tabular}{|c|c|c|c|}
\hline Variável & $\beta$ & EPB & $\mathrm{p}$ \\
\hline \multicolumn{4}{|l|}{ Grupo clínico } \\
\hline \multicolumn{4}{|c|}{ Comportamentos-problema infantis (CBCL) } \\
\hline Depressão (BDI) & 0,354 & 0,395 & 0,021 \\
\hline Estilos parentais (IEP) & 0,316 & 0,527 & 0,038 \\
\hline \multicolumn{4}{|c|}{ Comportamentos-problema infantis (CBCL) } \\
\hline Neuroticismo (BFP) & 0,161 & 5,043 & 0,370 \\
\hline Estilos parentais (IEP) & 0,354 & 0,491 & 0,053 \\
\hline \multicolumn{4}{|l|}{ Grupo controle } \\
\hline \multicolumn{4}{|c|}{ Comportamentos-problema infantis (CBCL) } \\
\hline Depressão (BDI) & 0,240 & 0,409 & 0,044 \\
\hline Estilos parentais (IEP) & 0,215 & 0,323 & 0,070 \\
\hline \multicolumn{4}{|c|}{ Comportamentos-problema infantis (CBCL) } \\
\hline Neuroticismo (BFP) & 0,242 & 2,001 & 0,047 \\
\hline Estilos parentais (IEP) & 0,223 & 0,415 & 0,067 \\
\hline
\end{tabular}

EPB: Erro padrão de b.

A Tabela 6, abaixo, mostra a análise dos estilos parentais separadamente. No GCL, o estilo de punição inconsistente media a relação entre o neuroticismo dos pais/cuidadores e os comportamentos-problema das crianças com doenças crônicas (teste Sobel=2,061; $p=0,039$ ). Além disso, a negligência dos pais/cuidadores media a relação entre os comportamentos-problema infantis e a depressão dos pais/cuidadores (teste Sobel=2,066; $p=0,038$ ) e entre o neuroticismo os pais/cuidadores e os comportamentos-problema das crianças (teste Sobel=2,138; $p=0,032$ ). 
Tabela 6: Mediação de Determinados Estilos Parentais entre o Ajustamento Psicológico Infantil e o Ajustamento Psicológico dos Pais/Cuidadores (Modelos Estatisticamente Significativos)

\begin{tabular}{llll}
\hline Variável & $\beta$ & EPB & $p$ \\
\hline $\begin{array}{l}\text { Grupo clínico } \\
\text { Comportamentos-problema infantis (CBCL) }\end{array}$ & & & \\
$\quad$ Depressão (BDI) & 0,283 & 0,525 & 0,061 \\
$\quad$ Negligência (IEP) & 0,403 & 1,523 & 0,009 \\
Comportamentos-problema infantis (CBCL) & & & \\
$\quad$ Neuroticismo (BFP) & 0,195 & 4,334 & 0,209 \\
$\quad$ Punição inconsistente (IEP) & 0,428 & 1,609 & 0,008 \\
Comportamentos-problema infantis (CBCL) & & & \\
$\quad$ Neuroticismo (BFP) & 0,182 & 4,337 & 0,239 \\
$\quad$ Negligência (IEP) & 0,443 & 1,589 & 0,006 \\
\hline $\begin{array}{l}\text { Grupo controle } \\
\text { Comportamentos-problema infantis (CBCL) }\end{array}$ & & & \\
$\quad$ Depressão (BDI) & 0,195 & 0,324 & 0,101 \\
$\quad$ Negligência (IEP) & 0,274 & 1,215 & 0,023 \\
Comportamentos-problema infantis (CBCL) & & 0,122 & 0,318 \\
Depressão (BDI) & 0,360 & 1,553 & 0,004 \\
Abuso físico (IEP) & & &
\end{tabular}

EPB: Erro padrão de b.

No GCO, a negligência dos pais media a relação entre os comportamentos-problema infantis e a depressão dos pais/ cuidadores (teste Sobel $=1,943 ; p=0,050$ ). Os abusos físicos cometidos pelos pais/cuidadores também mediam a relação entre os comportamentos-problema infantis e a depressão dos pais/cuidadores (teste Sobel=2,697; $p=0,006$ ).

\section{DISCUSSÃO}

Este estudo comparou o ajustamento psicológico de crianças e adolescentes com doenças crônicas com crianças e adolescentes sem este histórico, demonstrando que os pacientes que sofrem com enfermidades crônicas apresentam níveis similares de ajustamento psicológico que seus pares "saudáveis". Tal resultado refuta a primeira hipótese deste estudo, mas confirma os dados de Boekaerts e Röder (1999) e Ferguson e Walker (2012), que evidenciaram que os pacientes pediátricos crônicos não apresentam maior desajustamento psicológico, demonstrando baixos níveis de problemas externalizantes e internalizantes.

Também não foram detectadas diferenças significativas entre o grupo clínico e o grupo controle em relação ao ajusta- mento psicológico dos cuidadores, em discordância com a primeira hipótese deste estudo, mas em consonância com algumas pesquisas anteriores (Cohen, 1999; Nabors et al., 2013). Tais estudos sinalizam que, apesar de existirem grupos que estão em risco significativo de desajustamento psicológico, pode-se observar um ajustamento adequado em uma parcela considerável de famílias. Neste sentido, devem ser observados e pesquisados com maior profundidade fatores individuais relacionados a estas variações, como indicam Santos et al. (2013).

Os resultados aqui descritos são mantidos mesmo quando foram analisados somente os pais/cuidadores de pacientes com doenças mais graves, sugerindo que a severidade da doença tem pouca influência no ajustamento psicológico dos cuidadores, assim como encontrado por Fee e Hinton (2011). Apesar disto, não foi encontrada nenhuma pesquisa quantitativa brasileira nesta área de forma a estabelecer uma comparação mais confiável com os dados obtidos neste estudo.

Faz-se importante destacar que as duas fontes de informação sobre o ajustamento psicológico infantil utilizadas no presente estudo (cuidadores e crianças) mostraram-se significativamente relacionadas no GCL. Tais resultados indicam maior confiabilidade e menor viés no autorrelato infantil, refutando 


\section{MLE INTERACÃO EM LF PSICOLOGIA}

Amanda Muglia Wechsler, Denise Catricala Bution, Taís Galvão, Jaqueline Garcia Diniz e a afirmação de Phipps (2002) de que estes pacientes tenderiam a ocultar e/ou mascarar informações negativas sobre si mesmos. Por outro lado, não se encontrou relação significativa entre os autorrelatos das crianças do grupo controle e de seus pais/cuidadores. Pode-se hipotetizar que estes pais estejam menos atentos aos comportamentos de seus filhos do que os pais de crianças com doenças crônicas, visto que a condição médica leva, muitas vezes, a uma maior aproximação familiar (Oliveira et al., 2012).

Assim, o ajustamento psicológico adequado dos pacientes com doenças crônicas e de seus cuidadores demonstra que, apesar dos diversos estressores presentes em sua rotina, eles tendem a mostrar indicadores de resiliência. Portanto, os dados sugerem que não só as crianças com doenças crônicas apresentam relatos fidedignos sobre seus estados emocionais como também lidam com os desdobramentos da doença de forma resiliente, sendo este um padrão comum neste tipo de paciente, segundo Ferguson e Walker (2012).

Além disto, o ajustamento de pais/cuidadores e filhos demonstrou estar significativamente relacionado em ambos grupos, corroborando a segunda hipótese deste estudo e a literatura sobre o tema (Cousino \& Hazen, 2013; Jackson et al., 2015). Deste modo, de acordo com Cohen (1999), o ajustamento parental pode funcionar como um fator protetor no ajustamento psicológico infantil, ajudando as crianças a balancear as emoções e a ter maior repertório de respostas frente aos estressores da doença. No entanto, as análises realizadas no presente estudo não permitem afırmar a direção do efeito e sua causalidade, o que deve ser investigado em futuras pesquisas.

Não obstante, alguns subgrupos podem estar em risco psicológico. As análises mostraram que as crianças diagnosticadas com doenças crônicas há mais tempo apresentavam mais problemas de ajustamento, o que pode se relacionar a um maior tempo de tratamento, como constataram Poggi et al. (2005). Presume-se que um tratamento longo gere mais consequências físicas e psicológicas no paciente, prejudicando suas atividades diárias, relacionamentos interpessoais e rendimento acadêmico (Turkel \& Pao, 2007), impactando assim em seu ajustamento psicológico.

Outro resultado encontrado no GCL foi que os pacientes que possuíam pais/cuidadores com idades mais elevadas demonstraram maior desajustamento psicológico. Considerando que pais/cuidadores mais velhos possuem maiores expectativas parentais e de cuidado e menor senso de competência parental e menor responsividade (Ferreira et al., 2014; Östeberg \& Hagekull, 2000), é possível que haja um efeito spillover nos pacientes (Wechsler \& Rani, 2018). Contudo, mais pesquisas são necessárias para melhor compreensão desta variável.
Com relação às práticas parentais referidas, os grupos também não diferiram entre si, contrariando os achados de Piccinini et al. (2003). Este resultado, no entanto, é compreensível quando se considera a ausência de diferenças entre os grupos em relação ao ajustamento psicológico dos pais e crianças. Assim, parece que as práticas parentais tem mais uma função mediadora do que direta no ajustamento dos pacientes e de seus cuidadores; efeito este confirmado nas análises deste estudo. Deste modo, constatou-se que as práticas parentais negativas, principalmente a negligência e a punição inconsistente, exercem um papel mediador significativo no ajustamento das crianças com doenças crônicas e de seus cuidadores, corroborando a terceira hipótese deste estudo e os achados de Celano et al. (2008), Leinonen et al. (2003), Lim et al. (2008), Murphy et al. (2017) e Pinquart (2013). Por outro lado, não foi constatada tal função em relação às práticas parentais positivas.

Não obstante, faz-se necessário contextualizar os resultados obtidos a partir dos múltiplos estressores presentes nas vidas das famílias com crianças com doenças crônicas. Esta população está submetida a consultas médicas recorrentes, procedimentos invasivos, hospitalizações constantes e/ou prolongadas, possibilidade de morte, mudança de rotina alimentar e laboral, entre outros, que possam resultar em práticas parentais mais coercitivas, visto que os pais se sentem sobrecarregados e estafados com tantas demandas (Pinquart, 2013). Além disto, o baixo nível socioeconômico - presente nas amostras de ambos grupos deste estudo - pode amplificar os efeitos dos estressores próprios da enfermidade crônica, tais como maiores dificuldades de acesso à assistência médica e de provimento a necessidades específicas relacionadas à condição crônica (Moreira et al., 2017). Igualmente, outras variáveis presentes em famílias de baixa renda (violência e conflitos familiares, maior escassez de atividades adequadas de lazer, físicas, culturais e sociais), podem exercer influência nas práticas educativas observadas (Celano et al., 2008).

Todo este contexto pode influenciar tanto no ajustamento psicológico infantil (criando ou mantendo problemas de comportamento e stress crônico) como no ajustamento psicológico dos cuidadores, acarretando em menor responsividade dos pais/cuidadores e maior uso de punição (Evans \& Kim, 2013; Santos et al., 2013). Igualmente, outros fatores individuais dos pais/cuidadores - personalidade, psicopatologias pré-existentes - e sociais - rede de apoio social, relação conjugal, ocupação profissional -, não avaliados no presente estudo, também podem estar relacionados a práticas parentais negativas que, por sua vez, impactariam no ajustamento psicológico das crianças (Barroso \& Machado, 2015).

No entanto, resultados similares com relação ao papel mediador da negligência e de práticas punitivas parentais foram 
encontrados também no grupo controle, como hipotetizado, sugerindo que a condição crônica isoladamente não explique os problemas de ajustamento infantis, e que talvez estressores experimentados diariamente, tanto pelos pais como pelas crianças, sejam tão ou mais "traumáticos" e provoquem sensações mais intensas que a doença crônica em si, como discutido por Jurbergs et al. (2009). Além disto, o fato de cuidar de uma criança por si só é altamente estressante e a presença de uma doença crônica pode ter menos impacto do que outras variáveis inerentes ao processo de cuidar (Neece et al., 2012).

Portanto, a presente pesquisa contribui ao elucidar a influência do ajustamento parental e o papel mediador de práticas parentais negativas no processo de ajustamento psicológico de pacientes pediátricos com doenças crônicas. Dados os efeitos nocivos a curto e longo prazo de práticas coercitivas, ainda que estas não sejam exercidas de modo único e/ou contínuo (Piccini et al., 2003; Sidman, 1989/ 2003; Wechsler \& Rani, 2018), os resultados obtidos merecem atenção. Assim, o Ministério da Saúde (2010) alerta que crianças e adolescentes com doenças crônicas estão em alto risco para violência por parte de seus cuidadores, e enfatiza que ações preventivas e interventivas sejam adotadas com esta população.

No entanto, devido à natureza multidimensional, dinâmica e recíproca deste fenômeno, assim como o delineamento de pesquisa adotado, não é possível afirmar a direção dos efeitos observados, nem sua causalidade. Outras limitações deste estudo incluem a amostra reduzida e de conveniência, a utilização de instrumentos não específicos a pessoas com doenças crônicas e o uso de instrumentos de autorrelato com um único cuidador.

Tais problemas podem ser minimizados em futuras pesquisas observações naturalísticas de díades cuidadores-crianças, assim como o uso de metodologias experimentais. Além disto, seria importante a investigação de outros estressores associados à condição crônica e ao baixo nível socioeconômico, a ampliação do tamanho amostral, e a equiparação das amostras em relação ao sexo dos cuidadores, visto que podem haver diferenças nos estilos parentais em função desta variável. Finalmente, seria importante a coleta de medidas objetivas de saúde física dos pacientes e a comparação do ajustamento psicológico de grupos de crianças com diferentes doenças crônicas.

A escassez de pesquisas brasileiras sobre o ajustamento psicológico em crianças com doenças crônicas e, mais ainda, sobre o papel mediador dos estilos parentais, dificulta a comparação e generalização dos resultados. Da mesma forma, a inexistência de instrumentos psicológicos especificamente voltados para a avaliação desta população não permite a análise de aspectos fundamentais implícitos nesta condição médica. Portanto, urge o desenvolvimento e/ou adaptação de testes psicológicos direcionados a estes indivíduos.

Assim, os resultados obtidos com a presente pesquisa podem auxiliar as equipes de saúde na identificação de grupos em risco de desajustamento psicológico, visando estratégias de promoção de comportamentos saudáveis e o delineamento de intervenções psicológicas. Estas, por sua vez, podem propiciar uma melhora do funcionamento familiar e, consequentemente, melhor adaptação dos pacientes pediátricos à condição crônica.

Por último, cabe ressaltar a necessidade do cuidado psicológico aos cuidadores, visto que a maioria das práticas de psicólogos da saúde está concentrada nos pacientes pediátricos (Meyler et al., 2010). Deste modo, sugerem-se intervenções psicológicas direcionadas especificamente ao ensino e aprimoramento de práticas educativas parentais positivas, com o uso de modelação, modelagem e role-play em grupos de cuidadores, uma prática viável e adequada ao contexto brasileiro, promovendo o bem-estar, a qualidade de vida e a resiliência de famílias e pacientes com doenças crônicas.

\section{DECLARAÇÃO DA CONTRIBUIÇÃO DOS AUTORES:}

Certificamos que todos os autores participaram suficientemente do trabalho para tornar pública sua responsabilidade pelo conteúdo. A contribuição de cada autor pode ser atribuída como se segue:

A. M. W. contribuiu para a administração do projeto, pela conceitualização, análise dos dados e redação do artigo final.

D. C. B., T.G., J.D. e C.B.G. contribuíram para a conceitualização, investigação e revisão do artigo.

\section{DECLARAÇÃO DE CONFLITOS DE INTERESSE}

Os autores declaram que não há conflitos de interesse no manuscrito submetido

No texto do manuscrito suprimimos a seguinte informação para garantir o anonimato dos autores. Pedimos que essa informação seja incorporada ao texto na ocasião da sua diagramação

[Comitê de Ética do Centro Universitário Unifafibe inscrito na Plataforma Brasil sob o número: 30409214.5.0000.5387]

\section{AGRADECIMENTOS}

As autoras agradecem à Fundação Nacional de Desenvolvimento do Ensino Superior (Funadesp) pelo financiamento da pesquisa. 


\section{H MTERAC̄OOEM ET. PSICOLOGIA}

Amanda Muglia Wechsler, Denise Catricala Bution, Taís Galvão, Jaqueline Garcia Diniz e

Cristiane Bossi Gabriel

\section{REFERÊNCIAS}

Achenbach, T. M. \& Rescorla, L. A. (2007). Multicultural supplement to the manual for the ASEBA School-Age Forms \& Profiles. University of Vermont, Research Center for Children, Youths, \& Families.

Bai, G., Herten, M. H., Landgraf, J. M., Korfage, I. J., \& Raat, H. (2017). Childhood chronic conditions and health-related quality of life: Findings from a large population-based study. PLoS One, 12 (6), e0178539. https://doi.org/10.1371/ journal.pone.0178539

Baron, R. M. \& Kenny, D. A. (1986). The moderator-mediator variable distinction in Social Psychology research: conceptual, strategic, and statistical considerations. Journal of Personality and Social Psychology, 51(6), 1173-1182.

Barros, L., Matos, M.G., \& Batista-Foguet, J. (2008). Chronic diseases, Social context and adolescent health: Results of the Portuguese National Health Behaviour in School-Aged Children Survey. Revista Brasileira de Terapias Cognitivas, 4(1). https://doi.org/ 10.5935/1808-5687.20080003

Barroso, R. G. \& Machado, C. (2015). Definições, dimensões e determinantes da parentalidade. In: Pluciennik. G. A., Lazzari, M. C. \& Chicaro, M. F. (Orgs). O papel das práticas e estilos parentais no desenvolvimento da primeira infância (pp. 16-32). Fundação Maria Cecilia Souto Vidigal.

Celano, M., Bakeman, R., Gaytan, O., Smith, C. O., Koci, A., \& Henderson, S. (2008). Caregiver depressive symptoms and observed family interaction in low-income children with persistent asthma. Family Process, 47(1), 7-20. https://doi. org/10.1111/j.1545-5300.2008.00236.x

Cohen, M. S. (1999). Families coping with childhood chronic illness: a research review. Families System \& Health, 17: 149164. http://dx.doi.org/10.1037/h0089879

Compas, B. E., Jaser, S. S., Dunn, M. J., \& Rodríguez, E. M. (2012). Coping with chronic illness in childhood and adolescence. Annual Review of Clinical Psychology, 27(8), 455-480. https://doi.org/10.1146/annurev-clinpsy-032511-143108

Cousino, M. K. \& Hazen, R. A. (2013). Parenting stress among caregivers of children with chronic illness: a systematic review. Journal of Pediatric Psychology, 38(8), 809-828. https://doi.org/10.1016/j.pedn.2015.01.024

Cunha, J. A. (2001). Manual da versão em português das Escalas Beck. Casa do Psicólogo.

Ellis, D. A., Podolski, C. L., Frey, M., Naar-King, S., Wang, B., \& Moltz, K. (2007). The role of parental monitoring in adolescent health outcomes: Impact on regimen adherence in youth with type 1 diabetes. Journal of Pediatric Psychology, 32(8), 907-917. https://doi.org/0.1093/jpepsy/jsm009

Evans. G. W. \& Kim, P. (2013). Childhood poverty, chronic stress, self-regulation and coping. Child Development Perspectives, 7(1), 43-48. https://doi.org/10.1111/cdep.12013
Fee, R. J. \& Hinton, V. J. (2011). Resilience in children diagnosed with a chronic neuromuscular disorder. Journal of Developmental \& Behavioral Pediatrics, 32(9), 644-650. https:// doi.org/10.1097/DBP.0b013e318235d614

Ferreira, B., Monteiro, L., Fernandes, C., Cardoso, J., Veríssimo, M., \& Santos, A. J. (2014). Percepção de Competência Parental: Exploração de domínio geral de competência e domínios específicos de auto-eficácia, numa amostra de pais e mães portuguesas. Análise Psicológica, 32(2), 145-156. https://doi.org/10.14417/ap.854

Ferro, M. A. \& Boyle, M. H. (2013). Self-concept among youth with a chronic illness: a meta-analytic review. Health Psychology, 32(8), 839-848. https://doi.org/10.1037/a0031861

Ferro, M. A. \& Boyle, M. H. (2015). The impact of chronic physical illness, maternal depressive symptoms, family functioning, and self-esteem on symptoms of anxiety and depression in children. Journal of Abnormal Child Psychology, 43(1), 177-187. https://doi.org/10.1007/s10802-014-98936

Ferguson, P. \& Walker, H. (2012). "Getting on with life": resilience and normalcy in adolescents living with chronic illness. International Journal of Inclusive Education, 18(3), 227-240. https://doi.org/10.1080/13603116.2012.676082

Gomide, P. I. C. (2006). Inventário de Estilos Parentais. Vozes.

Holmbeck, G.N. (1997). Toward terminological, conceptual, and statistical clarity in the study of mediators and moderators: examples from the child-clinical and pediatric psychology literatures. Journal of Consulting and Clinical Psychology, 65(4), 599-610. https://doi.org/10.1037/ 0022-006X.65.4.599

Jackson, A. C., Frydenberg, E., Liang, R. P., Higgins, R. O., \& Murphy, B. M. (2015). Familial impact and coping with child heart disease: a systematic review. Pediatric Cardiology, 36(4), 695-712. https://doi.org/10.1007/s00246-015-1121-9

Jaser, S. S. \& Grey, M. (2010). A pilot study of observed parenting and adjustment in adolescents with type 1 diabetes and their mothers. Journal of Pediatric Psychology, 35(7), 738-747. https://doi.org/10.1093/jpepsy/jsp098

Jurbergs, N., Long, A. L., Ticona, L., \& Phipps, S. (2009). Symptoms of posttraumatic stress in parents of children with cancer: are they elevated relative to parents of healthy children? Journal of Pediatric Psychology, 34(1), 4-13. https:// doi.org/10.1093/jpepsy/jsm119

Leinonen, J. A., Solantaus, T. S., \& Punamäki, R. L. (2003). Parental mental health and children's adjustment: the quality of marital interactions and parenting as mediating factors. Journal of Child Psychology and Psychiatry, 44(2), 227-241. https://doi.org/10.1111/1469-7610.t01-1-00116

Lim, J. H., Wood, B. L., \& Miller, B. D. (2008). Maternal depression and parenting in relation to child internalizing symptoms and asthma disease activity. Journal of Family Psychology, 22(2), 264-273. https://doi.org/10.1037/08933200.22.2.264 
Lipp, M. E. N. \& Lucarelli, M. D. M. (2011). ESI: Escala de Stress Infantil - manual. 2a edição. Casa do Psicólogo.

Meyler, E., Guerin, S., Kiernan, G., \& Breatnach, F. (2010). Review of family-based psychosocial interventions for childhood cancer. Journal of Pediatric Psychology, 35, 1116-1132. https://doi.org/10.1093/jpepsy/jsq032

Ministério da Saúde (2010). Linha de cuidado para a atenção integral à saúde de crianças, adolescentes e suas famílias em situação de violências: orientação para gestores e profissionais de saúde. https://bvsms.saude.gov.br/bvs/publicacoes/linha_cuidado_atencao_integral_saude.pdf

Ministério da Saúde (2014). Pesquisa Nacional de Saúde 2013: percepção do estado de saúde, estilos de vida e doenças crônicas. Instituto Brasileiro de Geografia e Estatística. https://ftp.ibge.gov.br/PNS/2013.

Moreira, M. C. N., Albernaz, L. V., Sá, M. R. C., Correia, R. F., \& Tanabe, R. F. (2017). Recomendações para uma linha de cuidados para crianças e adolescentes com condições crônicas complexas de saúde. Cadernos de Saúde Pública, 33(11), e00189516. https://doi.org/10.1590/0102-311x00189516

Murphy, L. K., Murray, C. B., Compas, B. E., Gerhardt, C. A., Berg, C. A., Wiebe, D. J., \& Holmbeck, G. N. (2017). Topical Review: Integrating Findings on Direct Observation of Family Communication in Studies Comparing Pediatric Chronic Illness and Typically Developing Samples, Journal of Pediatric Psychology, 42(1) 85-94. https://doi.org/10.1093/ jpepsy/jsw051

Nabors, L. A. Kichler, J. C., Brassel, A., Thakkar, S., Bartz, J., Pangallo, J., Van Wassenhove, B., \& Lundy, H. (2013). Factors related to caregiver state anxiety and coping with a child's chronic illness. Families, System \& Health, 31(2), 171-180. https://doi.org/10.1037/a0031240

Neece, C. L., Green, S. A., \& Baker, B. L. (2012). Parenting stress and child behavior problems: A transactional relationship across time. American Journal on Intellectual and Developmental Disabilities, 117(1), 48-66. https://doi. org/10.1352/1944-7558-117.1.48

Nunes, C. H. S. S., Hutz, C. S., \& Nunes, M. F. O. (2013). BFP: Bateria Fatorial de Personalidade - manual. Casa do Psicólogo.

Oliveira, C., Araújo, B., \& Rodrigues, V. (2012). Famílias de crianças com doença crônica: Percepção de coesão familiar. Transferibilidade do conhecimento em enfermagem da família. Escola Superior de Enfermagem do Porto.

Olvera, N. \& Power, T. G. (2010). Brief report: Parenting styles and obesity in Mexican American children: A longitudinal study. Journal of Pediatric Psychology, 35(3), 243-249. https://doi.org/10.1093/jpepsy/jsp071
Organização Mundial da Saúde (2014). Integrating the response to mental health disorders and other chronic diseases in health care systems. World Health Organization. https:// www.who.int/publications-detail-redirect/9789241506793

Östberg, M. \& Hagekull, B. (2000). A structural modeling approach to the understanding of parenting stress. Journal of Clinical Child Psychology, 29(4), 615-625. https://doi. org/10.1207/S15374424JCCP2904_13

Phipps, S. (2002). Repressive adaptive style in children with chronic illness. Psychosomatic Medicine, 64(1), 34-42.

Piccinini, C. A., Castro, E. K., Alvarenga, P., Vargas, S., \& Oliveira, V. Z. (2003). A doença crônica orgânica na infância e as práticas educativas maternas. Estudos de Psicologia, 8(1), 75-83.

Pinquart, M. (2013). Do the parent-child relationship and parenting behaviors differ between families with a child with or without chronic illness? A meta-analysis. Journal of $\mathrm{Pe}$ diatric Psychology, 38(7), 708-721. https://doi.org/10.1093/ jpepsy/jst020

Pinquart, M. (2011). Behavior problems in children and adolescents with chronic physical illness: A meta-analysis. Journal of Pediatric Psychology, 36(9), 1003-1016. https://doi. org/10.1093/jpepsy/jsr042

Santos, T., Matos, M. G., Simões, M. C., Camacho, I., Tomé, G., \& Moreno Rodriguez, M. C. (2013). Estilos parentais e desenvolvimento positivo em crianças e adolescentes com doença crônica. Psicologia da Criança e do Adolescente, 4(2), 185-203.

Sidman, M. (2003). Coerção e suas implicações. (Trad. M. A. Andery \& T. M. Sério). Livro Pleno. (Originalmente publicado em 1989).

Sisto, F. F. \& Martinelli, S. C. (2004). Escala de autoconceito infanto-juvenil. Vetor.

Turkel, S. \& Pao, M. (2007). Late consequences of pediatric chronic illness. Psychiatry Clinics of North America, 30(4), 819-835. https://doi.org/10.1016/j.psc.2007.07.009

Wechsler, A. M. \& Rani, A. C. Z. (2018). Interacción familiar de madres e hijos brasileños: identificación de prácticas parentales de riesgo (pp. 15-22). In: A. C. Rios, C. R. Jara \& E. Savelza. (Orgs.). Familias y niñez: nuevas tensiones, nuevas respuestas. Mirada.

Data de submissão: 12/02/2019 Primeira decisão editorial: 05/11/2019 Aceite em 26/09/2020 